\section{$\underset{\substack{\text { hommes } \\ \text { \& migrations }}}{ }$}

\section{Hommes \& migrations}

Revue française de référence sur les dynamiques

migratoires

1305 | 2014

L'exil chilien en France

\title{
Lazare Ponticelli, immigré italien et dernier poilu français
}

\author{
Marie-Claude Blanc-Chaléard
}

\section{Q OpenEdition \\ Journals}

Édition électronique

URL : http://journals.openedition.org/hommesmigrations/2767

DOI : 10.4000/hommesmigrations.2767

ISSN : 2262-3353

Éditeur

Musée national de l'histoire de l'immigration

\section{Édition imprimée}

Date de publication : 1 janvier 2014

Pagination : 176-177

ISBN : 978-2919-040261

ISSN : $1142-852 X$

\section{Référence électronique}

Marie-Claude Blanc-Chaléard, « Lazare Ponticelli, immigré italien et dernier poilu français », Hommes \& migrations [En ligne], 1305 | 2014, mis en ligne le 11 juillet 2014, consulté le 22 septembre 2020. URL : http://journals.openedition.org/hommesmigrations/2767; DOI : https://doi.org/10.4000/ hommesmigrations.2767 


\section{LAZARE PONTICELLI IMMIGRÉ ITALIEN ET DERNIER POILU FRANÇAIS}

MARIE-CLAUDE BLANC-CHALÉARD, historienne, professeur à l'université de Paris Ouest-Nanterre-La Défense.

Le e hasard a voulu que le dernier poilu français fût un immigré italien. Lazare Ponticelli, né en 1897 en Emilie-Romagne, est entré dans l'histoire quelques années avant sa mort survenue le 12 mars 2008, à l'âge de cent dix ans. Ce hasard émerge en fait d'une histoire nationale qui, depuis deux siècles, s'est construite avec l'immigration et s'est nourrie des apports étrangers. Indispensables à la modernisation économique et au peuplement d'un pays menacé par la dépopulation pendant plus d'un demi-siècle, les étrangers ont été de tous les combats, au sens figuré comme au sens propre. Le déclenchement de la Première Guerre mondiale fut un moment emblématique. L'ordre de mobilisation à peine lancé, on vit les étrangers se presser devant les bureaux de recrutement improvisés par les associations, comme la Ligue franco-italienne. Beaucoup de ces volontaires étaient des ouvriers, mais ce sont des écrivains (I'Italien Ricciotto Canudo, le Belge Blaise Cendrars) qui rédigèrent l'appel placardé le 29 juillet 1914 dans les rues de Paris, repris dans la presse : "'heure est grave. Tout homme digne de ce nom doit aujourd'hui agir (...) Intellectuels, étudiants, ouvriers, hommes valides de toutes sortes - nés ailleurs, domiciliés ici - nous qui avons trouvé en France la nourriture de notre esprit ou la nourriture matérielle, groupons-nous en un faisceau solide de volontés mises au service de la plus grande France."

L'un des groupes les plus enthousiastes pour défendre la France, symbole de liberté et de démocratie face aux régimes autoritaires est celui des "Russes", en réalité les Juifs émigrés depuis les années 1880, fuyant l'Empire des Tsars et ses pogromes. Mais sur les 52 nationalités qui se pressèrent le 20 août 1914 pour les opérations de tri et d'incorporation sur la place des Invalides, les Italiens étaient les plus nombreux. A Paris, comme à Marseille, à Nice ou à Lyon, les bureaux furent submergés de demandes. Au total, on a compté 7125 engagés transalpins, sur un effectif de 29796 étrangers ; les "Russes" venant en second, au nombre de 2848 .

\section{Garibaldien sans l'être}

Ces combattants allogènes allaient être intégrés à la Légion étrangère. Celle-ci avait été créée en 1831 pour accueillir les étrangers désireux de s'engager dans l'armée française. La vocation de ses deux "Régiments étrangers" basés en Algérie était de combattre "hors du territoire continental du Royaume". La Première Guerre mondiale ouvre une nouvelle étape, avec la constitution de quatre régiments de marche, appelés à combattre aussi sur le continent. Décimés après un an de combat, les légionnaires seront regroupés en novembre 1915 dans le Régiment de marche de la Légion étrangère (RMLE), qui sera le plus décoré de France. Dès le 2 août 1914, Lazare Ponticelli se rend à la caserne de la Légion boulevard RichardLenoir et obtient son "bon pour engagement", en 
nance. De nombreuses associations d'émigrés se disaient alors "garibaldiennes", comme "la Lyre garibaldienne nogentaise", née en 1897 à Nogent, ville où vivait Lazare Ponticelli en 1914. Pourtant, d'après les nombreux témoignages qu'il a laissés, il semble bien que cette affectation à la Légion des garibaldiens ait été un effet du hasard, puisque pour lui, son unique volonté était de s'engager pour la France, "une manière de dire merci à ce pays qui mavait donné à manger". Le parcours de Lazare se profile très tôt comme celui d'un destin individuel, toujours engagé au sens humain du terme, mais pas au sens politique. II reste que son histoire, unique à bien des égards, est liée au destin collectif des montagnards italiens qui affluèrent en France à partir de la fin du XIXe siècle.

Lazare Ponticelli, en 2007, il avait alors 110 ans. (c) D.R.

mentant sur son âge. II n'a pas encore 17 ans. Le 22 septembre, il est transféré à Nîmes et affecté au $4^{\mathrm{e}}$ régiment de marche du $1^{\text {er }}$ Régiment étranger. Ce régiment n'est autre que celui resté dans I'histoire sous le nom de "Légion Garibaldi". Jusqu'à la médiatisation autour de Lazare Ponticelli, le récit de l'engagement italien aux côtés de la France en 1914 se résumait à la geste de cette "légion". Concession de l'Etat-major français, cette unité autonome, réclamée dès juillet 1914 par le fils Giuseppe Garibaldi, Ricciotti, qui avait combattu aux côtés de son père pour défendre la République française en 1870 , était composée d'Italiens à peu près exclusivement et commandée par un des petit-fils du "héros des deux mondes". On leur avait accordé le port de la chemise rouge. Le garibaldisme, très populaire en milieu immigré, était alors autant une forme d'extrême gauche ouvrière qu'une manifestation de l'amitié franco-italienne, voire de la double apparte-

\section{Itinéraire d'un enfant des Apennins émigré en France}

La grande émigration des Italiens a commencé dans les années 1880. Ceux du Mezzogiorno se ruent vers les Amériques, tandis que Piémontais, Toscans et autres originaires des régions du nord et de l'ouest gagnent la France. Traditionnellement, la frontière des Alpes favorise les circulations entre les deux "sœurs latines", et Nice et la Savoie ne sont françaises que depuis 1860 . La vague transalpine de la fin de siècle n'en est pas moins vécue comme une "invasion" dans le midi méditerranéen, avec des manifestations violentes comme à Marseille (1881) ou à AiguesMortes (1893). II est vrai qu'en 1900, 1 habitant sur 5 est italien dans les départements côtiers. À cette date, les originaires d'outremonts sont au premier rang des étrangers recensés en France, devant les Belges. Lazare Ponticelli est né à Bettola, dans le Val Nure, une vallée apennine au sud de Plaisance, en Émilie-Romagne. Alors que Piémontais et Ligures dominent dans le Midi, les originaires de Parme et Plaisance sont nombreux 


\section{CHRONIQUES DE GUERRE}

en région parisienne. En banlieue, la commune de Nogent-sur-Marne se singularise dès les années 1870 avec une petite colonie de paysans du Val Nure, employés à la reconstruction du viaduc détruit par la guerre. La colonie grandit rapidement et dépasse les 500 personnes quand la mère de Lazare la rejoint en 1903. Elle a quitté le hameau et le maigre lopin familial après la mort du père, émigrant avec cinq de ses enfants, mais abandonnant le plus jeune, le petit Lazare, à des fermiers qui l'emploient comme berger. Épisode violent, mais à peine insolite dans l'Italie de l'époque. Pour bien des contadini (paysans) et bracchianti (journaliers), le nouvel État unifié n'apparaît pas comme une nation, mais comme la cause d'une misère aggravée. La modernisation va avec la crise agraire qui fait rage. La solution, c'est l'émigration, des hommes comme des femmes, mais les enfants inaptes au travail sont laissés au pays (le travail des enfants de moins de douze ans est interdit en France comme en Italie). Guère plus insolite est la figure de l'enfant tôt adulte qui prend en main son destin : Lazare économise pour payer son voyage, bricole lui-même les sabots qui lui permettront d'aller à pied jusqu'à la gare de Piacenza . II a neuf ans quand il débarque à la gare de Lyon avec pour tout viatique un papier où est inscrit le nom d'un café d'originaires qu'il ne trouve d'ailleurs pas. L'image de l'enfant en pleurs sur un banc de la gare de Lyon rappelle celui de Taravella, arrivé à treize ans en 1897 avec une adresse sur carton épinglé à sa veste. II deviendra l'un des entrepreneurs du bâtiment les plus importants de Nogent, associé à son cousin Cavanna. Lazare finit par rejoindre ses frères à Nogent, et partage la vie misérable des maçons et garçons-maçons italiens, et de leurs épouses et sœurs, blanchisseuses ou plumassières, relégués dans les vieilles ruelles du centre-ville. Avec la Belle Époque, les

Les bottes du soldat Lazare Ponticelli (C) MUSÉE DE L'HISTOIRE ET DES CULTURES DE L'IMMIGRATION

"macaronis" bruyants et "joueurs de couteaux" sont mieux tolérés qu'à la fin du siècle. Le marché du travail est plus ouvert, les grandes industries ont besoin d'eux, comme en Lorraine où se développe la sidérurgie. Dans d'autres activités comme le bâtiment, leur savoir-faire et leur courage physique sont appréciés, autant que leur frugalité. De petites affaires se montent. C'est ainsi que Lazare, le "petit Italien" des rues parisiennes, tour à tour ramoneur et crieur de journaux, se met à son compte à seize ans à peine et crée avec un associé une petite entreprise de ramonage de cheminées. Un an plus tard, c'est la déclaration de guerre et son associé est mobilisé. Alors que les rues résonnent de l'idée de défendre la France, Lazare se décide vite : qu'a-t-il à regretter de l'ttalie ? Celle-ci, alliée de l'Allemagne, reste d'ailleurs neutre. Son avenir à lui est ici et, pour que les affaires reprennent, il faut que la France gagne. Le bruit court aussi que les engagés auront la naturalisation gratuite. 


\section{Blessures de guerre}

Jusqu'aux premiers jours de novembre 1914, le régiment de Lazare Ponticelli est en garnison dans le Midi. Il quitte Nîmes le 13 décembre et rejoint un camp en Champagne pour quelques jours d'instruction sommaire: "Je ne savais pas vraiment tirer". Et pourtant, le 22 décembre, c'est le front de l'Argonne et très vite les terribles combats du bois de Bolante. Plus qu'à tirer sur l'ennemi, Lazare s'illustre par sa bravoure à sauver des vies, aidant son frère Céleste blessé aussi bien qu'un soldat allemand qu'il accompagne vers ses lignes. Ce tout jeune homme se trouve ainsi plongé dans I'hallucinante boucherie des tranchées. En deux semaines, la Légion garibaldienne perd un quart de son effectif combattant avec 93 tués, dont deux frères Garibaldi, 337 blessés et 136 disparus. Rapidement, l'unité est retirée du front, ramenée à la mi-mars à Avignon pour être démobilisée. II faut dire que les récits des engagés risquent de décourager au moment où les alliés font pression pour une entrée en guerre de l'Italie à leurs côtés. La légion est dissoute le 17 mars 1915, "I'Intervention" est décidée le 20 mai. Les légionnaires sont dans leur majorité affectés à l'armée italienne, certains de force, comme Lazare Ponticelli qui avait demandé à rester dans la Légion: "Moi, je voulais rester un soldat français. J'ai essayé de m'échapper, mais j'ai été emmené de force jusqu'à Turin par deux gendarmes qui m'ont laissé en me souhaitant bonne chance." Envoyé sur le front autrichien du Tyrol avec premier contingent d'Alpini (chasseurs alpins), il y fait une fois de plus l'expérience des horreurs et de l'absurdité de la guerre. Face à des soldats qui ne sont autres que des Italiens d'Autriche, qui parlent la même langue, Lazare participe activement au mouvement de fraternisation qui aboutit au refus de combattre. II échappe au conseil de guerre, mais est affecté dans une zone plus dangereuse, au Monte Cucco en Slovénie, où il s'illustre par de nouveaux exploits et où il est gravement blessé.

Lazare sort de la guerre avec des blessures plus profondes : "La guerre est une honte". Solidaire de tous les combattants, il refuse les distinctions qu'il reçoit à la fin de sa vie, simplement parce qu'il a survécu. Pourtant, il dit s'être senti heureux d'avoir contribué à la victoire et ne renie jamais son choix d'engagement pour la France. En 1939, il sera à nouveau prêt à combattre. Ébranlé par la violence de guerre, Lazare s'est donc montré également résistant dans ses convictions. Dès sa démobilisation en 1920, il rejoint la France, avec le louis d'or qu'on lui a donné pour prendre le train. Partout dans le monde, la guerre a violemment saisi les milieux d'émigrants, avec des réactions variables selon les individus. Certains ont cherché à se soustraire à la conscription en 1915, alimentant une suspicion durable en Italie à l'égard des émigrés. Les historiens ont récemment montré combien, pour la plupart, la guerre a été l'occasion de s'identifier à la patrie italienne dont ils avaient commencé, au loin, à saisir la présence. Des combats vint la rupture, définitive pour les morts ou les invalides. Patriotisme des tranchées, pacifisme ou révolte, les survivants ont été ébranlés, la fragile nation italienne aussi et la vague des émigrants des années 1920 porte la marque de cet ébranlement. Si l'idée de l'Italie parle davantage aux Italiens d'entre-deux-guerres qu'à ceux d'avant-guerre, elle est vite confisquée par le fascisme qui déferle dès 1920 pour s'installer au pouvoir en 1922. Pour beaucoup, la nouvelle émigration a les couleurs de l'exil.

\section{Retour en France}

Régions vitales dévastées, population masculine décimée, la France d'après-guerre voit plus que jamais son avenir lié à l'immigration. Le 


\section{CHRONIQUES DE GUERRE}

recrutement de travailleurs est organisé au loin, comme en Pologne. Après l'adoption des quotas aux États-Unis en 1920 et 1924, le pays devient la destination privilégiée des réfugiés de la Grande Guerre, comme des bannis de Mussolini. Même si désormais, les contrats de travail sont obligatoires, les voies traditionnelles de la migration restent ouvertes et bien des Italiens les empruntent. À peine plus nombreux qu'avant guerre en 1921 (451 000 recensés), ils sont plus de 800000 dix ans plus tard, représentant un tiers de l'énorme afflux migratoire des années 1920. À la différence de ce que vivent bien des nouveaux venus, le retour de Lazare Ponticelli s'inscrit alors dans une histoire migratoire qui a un passé. Migrant plein d'initiative dès ses débuts avant 14, il revient dans un pays où les potentialités sont immenses. En 1921, il crée, avec ses frères, une entreprise de fumisterie qui se spécialise dans le montage des cheminées d'usine, et s'installe au Kremlin Bicêtre. Autant que le sens des affaires, il montre toujours le même sens de l'humain et le même courage, devançant les ouvriers dans les tâches les plus rudes. Son entreprise prospère. Marié à Clara, une Française, en 1923, il sera père de trois enfants. Pour autant, comme souvent, la reconnaissance du pays d'accueil se fait attendre et la naturalisation ne vient qu'en 1939, moment stratégique où l'on naturalise massivement les Italiens. Jugé trop vieux pour combattre, il est affecté en zone sud avec son entreprise, et participe à la Résistance. La société Ponticelli frères compte aujourd'hui plus de quatre mille salariés en France et à l'étranger. Lazare voyait dans cette réussite l'issue de son plus grand combat. Une histoire exemplaire ? Certes, mais comme celles de milliers d'immigrés dont nous ignorons le parcours souvent incroyable. Tout comme demeure dans l'ombre l'héroïsme de la plupart des 8,4 millions de poilus de la Grande Guerre ou des 5,5 millions de soldats italiens, parmi lesquels les 5000 morts de la brigade Alpi à Bligny, dans la dernière offensive de Champagne, les 15 et 16 juillet 1918 . 